\title{
PROPORÇÃO DE CESARIANAS SEGUNDO COR DA PELE E ESCOLARIDADE MATERNA NAS DIFERENTES REGIÕES DO BRASIL: TENDÊNCIA TEMPORAL DE 2007 A 2016
}

\section{CESAREAN SECTIONS' PROPORTION ACCORDING TO SKIN COLOR AND MATERNAL SCHOOLING IN THE DIFFERENT REGIONS OF BRAZIL: TEMPORAL TREND FROM 2007 TO 2016}

\author{
Joyce Ribeiro Rothstein ${ }^{1}$ \\ Ana Caroline Secco ${ }^{2}$ \\ Rafaela Souza ${ }^{3}$ \\ Larissa Weber ${ }^{4}$ \\ Eleonora d'Orsi ${ }^{5}$ \\ Ana Luiza Curi Halla ${ }^{6}$ \\ Flávia Soares $^{7}$
}

Resumo: Este estudo ecológico de série temporal objetivou descrever a proporção de cesarianas segundo cor da pele e escolaridade maternas nas diferentes regiões do Brasil de 2007 a 2016. Os dados foram coletados no Sistema de Informação sobre Nascidos Vivos e a proporção de cesarianas segundo cor da pele e escolaridade materna foram observadas segundo as macrorregiões. Observou-se aumento na proporção de cesarianas até o ano de 2014 (57,07\%), com discreta diminuição nos anos de 2015 (55,55\%) e 2016 (55,44\%). Em todas as regiões, quanto maior o nível de escolaridade, maior a proporção de cesarianas. Em relação à cor da pele, a branca correspondeu a maior proporção em todas as regiões, especialmente na Centro Oeste e Sul. A indígena correspondeu à menor proporção de cesarianas, apesar de haver aumento principalmente no Sul, apontando para uma determinação da região de residência da mãe superior às demais variáveis como cor da pele e escolaridade.

Palavras-chave: Cesárea; escolaridade; grupos raciais; distribuição temporal.

Abstract: This ecological time-series study aimed to describe the proportion of cesarean sections according to skin color and maternal schooling in the different regions of Brazil from 2007 to 2016. Data were collected from the Brazil Live Birth Information System and the proportion of cesarean sections was analyzed according to skin color and maternal schooling, according to macro-regions. There was an increase in the proportion of cesareans until 2014 (57.07\%), with a slight decrease in the years 2015 (55.55\%) and 2016 (55.44\%). In all regions, the higher the educational level, the greater the proportion of cesarean sections. Regarding skin color, white presented the highest proportion in all regions, especially in the Midwest and South regions. The indigenous population had the lowest proportion of cesareans,

\footnotetext{
1Discente do Programa de Pós-Graduação em Saúde Coletiva, Doutorado, UFSC, Brasil. E-mail: joycefisio@gmail.com.

'Discente do Programa de Pós-Graduação em Saúde Coletiva, Doutorado, UFSC, Brasil. E-mail: ana.caroline.secco@gmail.com.

${ }^{3}$ Discente do Programa de Pós-Graduação em Saúde Coletiva, Doutorado, UFSC. Brasil. E-mail: rafa_nutri@yahoo.com.br.

${ }^{4}$ Discente do Programa de Pós-Graduação em Saúde Coletiva, Doutorado, UFSC, Brasil. E-mail: larissaweber1984@gmail.com.

${ }^{5}$ Docente do Programa de Pós-Graduação em Saúde Coletiva, Doutorado, UFSC, Brasil. E-mail: eleonora.dorsi@ufsc.br.

Docente do Programa de Pós-Graduação em Saúde Coletiva, Doutorado, UFSC, Brasil. E-mail: anacuri@gmail.com.

${ }^{7}$ Discente do Programa de Pós-Graduação em Saúde Coletiva, Mestrado, UFSC, Brasil. E-mail: flavia.pellegrin@gmail.com.
} 
despite an increase mainly in the South, pointing to a determination of the region of residence of the mother superior to the other variables, such as race and schooling.

Keywords: Cesarean section; educational status; continental population groups; temporal distribution.

\section{INTRODUÇÂO}

A cesariana é um procedimento cirúrgico indicado em caso de complicações na gravidez ou durante o parto. Evidências científicas apontam que a cesariana está associada a riscos maternos e perinatais, havendo seis vezes mais chances de complicações graves para a mãe em comparação ao parto normal, especialmente quando realizadas sem indicação (COSTA-RAMÓN et al., 2018; RATTNER; MOURA, 2016; BETRÁN et al., 2016; RYAN; NICHOLSON; MORRISON, 2018; WHO, 2010).

O aumento das cesarianas é um problema de abrangência global e suas taxas têm ultrapassado os limites aceitáveis em várias regiões do mundo (BARROS et al., 1991; BETRÁN et al., 2016; BOERMA et al., 2018). A Organização Mundial da Saúde (OMS) considera como taxa ideal de cesáreas entre 10\% e 15\% do total de partos, contudo a tarefa de definir o número adequado em termos populacionais permanece um grande desafio (ORGANIZAÇÃO MUNDIAL DA SAÚDE, 2015).

Há uma grande disparidade nas taxas de cesariana analisadas globalmente, alertando que em países pobres taxas próximas a zero são indicadores de falta de assistência e aumento de mortalidade materna-fetal. Por outro lado, em países de maior renda, as taxas elevadas podem indicar assistência pré-natal inadequada devido ao aumento da morbidade materna e neonatal (BOERMA et al., 2018).

No Brasil, observa-se a elevação progressiva das taxas de cesariana desde que foi iniciado o monitoramento dos dados. Em 2017 o país passou a ocupar a 2ª posição no ranking mundial, com um percentual de $57 \%$ de cesarianas. Dessa forma, $40 \%$ dos partos realizados na rede pública e $84 \%$ dos partos ocorridos na rede particular correspondem a cesarianas (RATTNER; MOURA, 2016; UNICEF, 2017).

De acordo com a UNICEF (2017), o elevado número de cesarianas realizadas antes do trabalho de parto está associado com maior risco de internações em UTI neonatal, presença de problemas respiratórios, risco de mortalidade e déficit de crescimento, quando comparados com crianças nascidas com idade gestacional entre 39 e 40 semanas. O maior risco decorre do fato de que as últimas semanas de gestação permitem maior ganho de peso, maturidade cerebral e pulmonar.

A realização de cesarianas, em números superiores aos indicados, tem razão multifatorial que contempla o estilo de prática dos profissionais da área: características maternas, fatores socioeconômicos, organizacionais, sociais e culturais (BETRÁN et al., 2016). Nesse contexto, as desigualdades sociais influenciam no risco de ocorrência de cesariana para mulheres do setor privado, quando comparadas à mulheres que tiveram parto nas maternidades públicas. As características que 
aumentam a probabilidade de ocorrência de cesariana no setor privado são: primíparas, mulheres de cor branca, o número maior de frequência em exame prénatal, parto diurno e história de natimorto em gestação anterior (FREITAS; FERNANDES, 2016).

A fim de compreender o fenômeno do aumento das cesarianas no Brasil e promover subsídios que possam modificar o cenário de medicalização do parto e suas consequências, o presente estudo tem como objetivo investigar a proporção de cesarianas, segundo cor da pele (raça/etnia) e escolaridade maternas nas diferentes regiões do Brasil, no período de 2007 a 2016.

\section{MÉTODOS}

Estudo ecológico descritivo de série temporal. As informações foram obtidas no mês de outubro de 2018, a partir do Sistema de Informação sobre Nascidos Vivos (SINASC) do banco de dados do DATASUS do Ministério da Saúde. O SINASC tem por objetivo reunir informações relativas aos nascimentos ocorridos em todo o território nacional, tendo como fonte dos dados a Declaração de Nascido Vivo (DN), a qual é padronizada e conta com cerca de 41 variáveis (SANTA CATARINA, 2018; BRASIL, 2018).

Foram selecionadas informações referentes aos anos de 2007 a 2016 sobre o evento "tipo de parto" e variáveis sociodemográficas maternas: cor da pele e escolaridade. Os dados foram levantados segundo as macrorregiões do Brasil, categorizadas da seguinte forma: Norte, Nordeste, Centro-Oeste, Sudeste e Sul. As variáveis sobre a cor da pele foram estratificadas de acordo com a classificação do Instituto Brasileiro de Geografia e Estatística (IBGE, 2011) em indígena, parda, preta, amarela e branca. Referente à escolaridade, a categorização foi apresentada da seguinte forma: nenhuma escolaridade, de 1 a 3 anos, de 4 a 7 anos, de 8 a 11 anos e 12 anos ou mais.

Os dados provenientes do DATASUS foram exportados para o Microsoft Excel® 2010, onde foram calculadas as proporções de cesarianas segundo a cor da pele e escolaridade por região do país. Os resultados foram transpostos para gráficos e tabelas no Excel.

A proporção de cesarianas por região (Norte, Nordeste, Sudeste, Sul, CentroOeste) foi calculada dividindo-se o número de cesarianas realizadas pelo número total de partos em cada região, multiplicado por 100. O cálculo foi feito para cada ano da série histórica.

A proporção de cesarianas por região segundo a escolaridade foi calculada dividindo-se o número de cesarianas realizadas em cada segmento (sem escolaridade, 1 a 3 anos, 4 a 7 anos, 8 a 11 anos e acima de 12 anos de estudo) pelo 
número total de partos nesses mesmos segmentos, em cada região, multiplicando o resultado por 100. O cálculo foi realizado ano a ano.

A proporção de cesarianas por região segundo a cor da pele foi calculada dividindo-se o número de cesarianas de acordo com cada região e cor da pele de interesse (branca, parda, preta, amarela e indígena) pelo número total de partos na mesma região e cor da pele, multiplicando o resultado por 100. Repetiu-se a fórmula para cada ano de interesse da série histórica.

\section{RESULTADOS}

No período estudado, foram registrados 29.147.308 partos no Brasil. Destes, 15.482.218 foram cesarianas, o que correspondeu a 53,11\% do total de partos realizados. Observou-se um aumento gradativo na proporção de cesarianas de 2007 $(46,56 \%)$ até $2014(57,07 \%)$, ano a partir do qual houve pela primeira vez um decréscimo (Figura 1).

Figura 1 - Proporção de cesarianas realizadas no Brasil de 2007 a 2016.

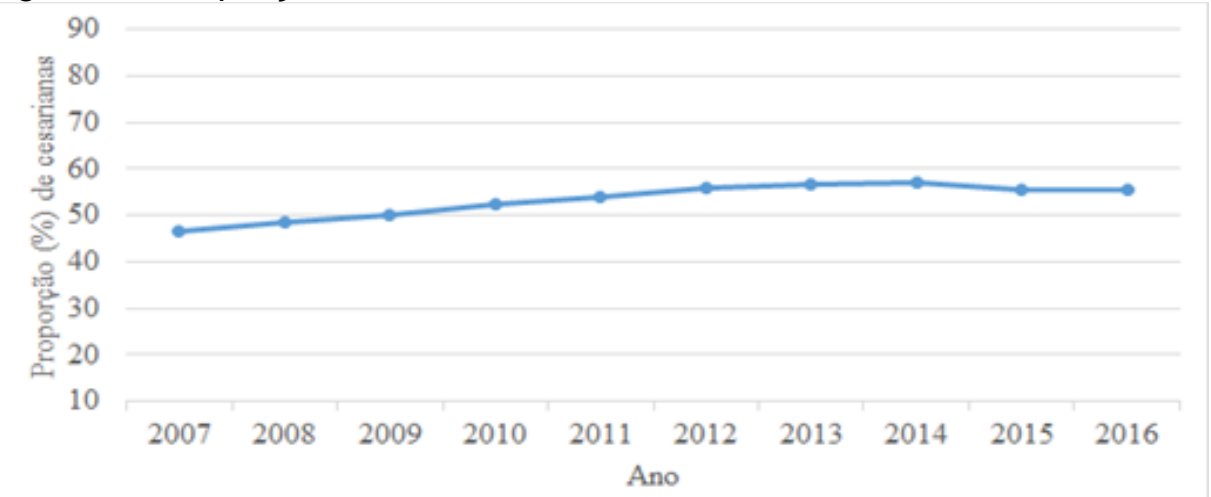

Fonte: Elaborado pelas pesquisadoras a partir de dados do Brasil, 2018.

Ao analisar a distribuição das cesarianas por região do país, verifica-se que as maiores proporções estão localizadas na região Centro-Oeste, seguida das regiões Sul e Sudeste (figura 2). A maior proporção de cesarianas ocorreu na região CentroOeste no ano de 2014 (61,81\%) e a menor na região Norte em 2007 (35,35\%). 
Figura 2 - Proporção de cesarianas segundo região do país de 2007 a 2016. 90

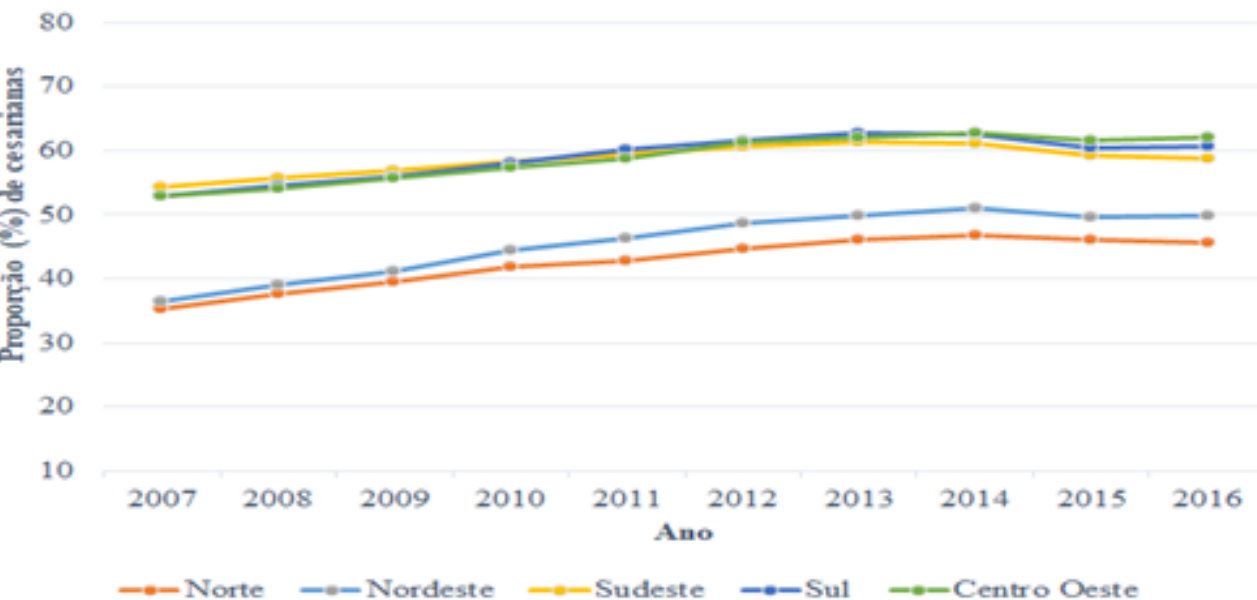

Fonte: Elaborado pelas pesquisadoras a partir de dados do Brasil, 2018.

\subsection{Proporção de cesarianas e escolaridade materna}

Os dados referentes à proporção de cesarianas e escolaridade materna estão apresentados na tabela 1.

Tabela 1 - Proporção de cesarianas segundo escolaridade da mãe e região do país de 2007 a 2016.

\begin{tabular}{|c|c|c|c|c|c|c|c|c|c|c|}
\hline \multirow[b]{2}{*}{ Escolaridade } & \multicolumn{10}{|c|}{ Ano } \\
\hline & 2007 & 2008 & 2009 & 2010 & 2011 & 2012 & 2013 & 2014 & 2015 & 2016 \\
\hline \multicolumn{11}{|l|}{ Norte } \\
\hline Nenhuma & 14,97 & 14,65 & 14,98 & 15,19 & 15,74 & 15,86 & 16,24 & 15,67 & 17,94 & 15,41 \\
\hline 1 a 3 anos & 20,94 & 22,72 & 23,42 & 26,20 & 25,53 & 26,49 & 26,48 & 27,36 & 27,21 & 27,54 \\
\hline 4 a 7 anos & 27,73 & 29,63 & 30,55 & 32,00 & 32,79 & 33,77 & 34,86 & 34,77 & 34,57 & 34,16 \\
\hline 8 a 11 anos & 42,82 & 43,88 & 45,56 & 46,91 & 46,82 & 48,74 & 49,18 & 49,40 & 48,00 & 46,51 \\
\hline 12 anos e mais & 62,31 & 64,69 & 66,66 & 67,73 & 74,83 & 76,80 & 77,08 & 76,24 & 74,15 & 73,08 \\
\hline \multicolumn{11}{|l|}{ Nordeste } \\
\hline Nenhuma & 17,14 & 18,92 & 20,79 & 22,70 & 24,52 & 25,79 & 26,31 & 28,47 & 26,10 & 28,50 \\
\hline 1 a 3 anos & 24,16 & 26,67 & 27,89 & 30,38 & 30,94 & 32,12 & 32,75 & 33,95 & 33,33 & 33,88 \\
\hline 4 a 7 anos & 28,85 & 30,90 & 31,97 & 34,36 & 35,64 & 37,28 & 37,95 & 39,08 & 37,72 & 38,37 \\
\hline 8 a 11 anos & 42,59 & 44,31 & 45,95 & 48,37 & 49,69 & 51,05 & 51,69 & 51,95 & 50,01 & 49,84 \\
\hline 12 anos e mais & 63,56 & 64,84 & 66,60 & 69,55 & 78,63 & 82,16 & 82,31 & 81,65 & 79,03 & 77,76 \\
\hline \multicolumn{11}{|l|}{ Sudeste } \\
\hline Nenhuma & 32,98 & 33,09 & 35,1 & 36,95 & 37,64 & 38,56 & 41,04 & 41,9 & 41,01 & 41,74 \\
\hline 1 a 3 anos & 40,78 & 41,26 & 41,61 & 42,97 & 44,63 & 45,76 & 46,42 & 46,54 & 45,35 & 44,37 \\
\hline 4 a 7 anos & 41,49 & 42,7 & 43,18 & 43,9 & 45,06 & 46,44 & 46,85 & 46,89 & 45,31 & 44,56 \\
\hline 8 a 11 anos & 54,78 & 55,69 & 56,17 & 57,06 & 57,71 & 58,49 & 58,64 & 57,8 & 55,55 & 54,96 \\
\hline 12 anos e mais & 75,79 & 77,04 & 77,81 & 79,03 & 81,74 & 84,21 & 84,43 & 82,94 & 79,78 & 78,82 \\
\hline \multicolumn{11}{|l|}{$\underline{\text { Sul }}$} \\
\hline Nenhuma & 32,98 & 33,45 & 33,71 & 34,91 & 38,28 & 39,75 & 41,19 & 38,54 & 38,83 & 42,7 \\
\hline 1 a 3 anos & 37,02 & 38,97 & 39,25 & 41,66 & 43,16 & 44,29 & 46,42 & 45,67 & 43,08 & 44,01 \\
\hline 4 a 7 anos & 40,39 & 41,83 & 42,57 & 43,88 & 45,32 & 47,11 & 47,74 & 47,13 & 45,16 & 45,31 \\
\hline 8 a 11 anos & 54,34 & 55,25 & 56,07 & 57,74 & 59,55 & 60,39 & 60,96 & 60,33 & 57,72 & 57,35 \\
\hline 12 anos e mais & 75,2 & 75,58 & 76,97 & 78,48 & 81,67 & 85,02 & 84,86 & 83,47 & 80,56 & 79,77 \\
\hline \multicolumn{11}{|l|}{ Certro Oeste } \\
\hline Nenluma & 24,36 & 28,6 & 28,31 & 28,91 & 26,7 & 26,92 & 25,23 & 31,46 & 27,85 & 28,06 \\
\hline 1 a 3 anos & 39,21 & 39,97 & 38,25 & 35,47 & 40,41 & 41,05 & 42,33 & 43,63 & 43,77 & 41,5 \\
\hline 4 a 7 anos & 40,26 & 41,03 & 42,56 & 44,16 & 44,94 & 47,26 & 47,99 & 47,54 & 46,17 & 46,59 \\
\hline 8 a 11 anos & 53,93 & 54,67 & 55,95 & 57,01 & 57,16 & 59,19 & 59,5 & 60,23 & 58,4 & 58,82 \\
\hline 12 anos e mais & 76,76 & 77,32 & 78,45 & 79,99 & 82,49 & 85,65 & 85,14 & 83,46 & 80,99 & 80,42 \\
\hline
\end{tabular}

Fonte: Elaborado pelas pesquisadoras a partir de dados do Brasil, 2018. 
Verificou-se em todas as regiões que quanto maior o nível de escolaridade, maior a proporção de cesarianas realizadas. As mulheres sem nenhuma escolaridade nas regiões sudeste e sul fazem cerca de 1,5 a 2,5 vezes mais cesarianas quando comparadas às mulheres, nesta mesma categoria de escolaridade, nas regiões Norte e Nordeste.

Houve uma tendência de crescimento gradual nas proporções de cesarianas em todas as regiões do país em todas as escolaridades de 2007 até 2013, ano a partir do qual a proporção de cesarianas na categoria de mulheres com 12 ou mais anos de estudos começou a diminuir. Na região Centro-Oeste, esse decréscimo já pode ser observado a partir de 2012, após ser registrada a maior proporção de cesarianas no país $(85,65 \%)$.

No ano 2016, ocorreu um aumento na proporção de cesarianas nas mulheres sem nenhuma escolaridade em todas as regiões, exceto na região Norte. Quando analisadas individualmente cada região do país, ganham expressão algumas variações ao longo do período. A região Norte destaca-se por ser a única onde a proporção de cesarianas entre as mulheres de maior escolaridade não atingiu a faixa dos $80 \%$. A maior proporção foi apresentada em 2013 , totalizando $77,08 \%$ dos partos realizados.

Na região Nordeste, verificou-se que a partir de 2014 houve diminuição nas cesarianas em todos os níveis de escolaridade; porém já no ano seguinte, a taxa voltou a aumentar entre as mulheres com menor ou sem escolaridade. Ainda na região Nordeste, no último ano analisado, pode-se observar que as mulheres com 12 anos ou mais de estudo apresentaram uma diferença de $27,96 \%$ em relação às mulheres com 8 a 11 anos de estudo, as quais corresponderam a segunda maior proporção de cesarianas. Dessa forma, a região Nordeste apresentou a maior diferença entre as duas categorias de mulheres com maiores escolaridades.

$\mathrm{Na}$ região Sudeste, foi observado aumento gradativo na proporção de cesarianas até o ano de 2013 para mulheres com 8 a 11 e mais de 12 anos de estudo. As demais categorias apresentaram aumento nas taxas até o ano de 2014. Nos anos seguintes observou-se uma queda nas proporções em todas as categorias, com exceção das mulheres sem nenhuma escolaridade, que apresentaram leve aumento na taxa, atingindo $41,75 \%$ em 2016. Interessante destacar que, nesta região, as categorias com baixa escolaridade ( 1 a 3 anos e 4 a 7 anos de estudo) tiveram suas taxas praticamente sobrepostas ao longo da série histórica.

Ao analisar a região Sul, percebe-se oscilações ao longo da série histórica em todas as categorias. No ano de 2016, houve uma aproximação entre as taxas nas categorias com menor e sem nenhuma escolaridade. Dentre as regiões analisadas, a região Centro-Oeste foi a que mais apresentou oscilações nos anos observados. No ano de 2016, a proporção de cesarianas manteve-se maior quando comparado com o ano de 2007 em todos os níveis de escolaridade. 


\subsection{Proporção de cesarianas segundo a cor da pele materna}

Na proporção de cesarianas segundo a cor da pele (tabela 2), observou-se que a indígena corresponde a menor e a branca a maior proporção de cesarianas em todas as regiões do país. As mulheres classificadas como indígenas nas regiões sudeste e sul fazem cerca de 3 a 4 vezes mais cesarianas quando comparadas às indígenas da região norte.

Nas regiões Norte e Nordeste, houve elevação na proporção em todas as classificações, com destaque para a amarela, que apresentou oscilações ao longo da série histórica, passando de 32,73\% (2007) para 56,81\% (2016) na região Norte e de 18,86\% (2007) para 51,09\% (2016) na região Nordeste. Já a região Sudeste foi uma das regiões que menos apresentou oscilações entre os anos estudados. A branca correspondeu a maior proporção de cesarianas em 2016 (67,38\%) e a indígena, a menor (35,36\%).

Na região Sul, as mulheres brancas finalizaram a série histórica com a maior proporção de cesarianas, compreendendo $62,85 \%$. Já a amarela manteve-se com a maior proporção de 2007 (60,62\%) até 2014 (65,06\%), caindo para 58,54\% em 2016. A indígena, apesar de apresentar aumento, representou a menor proporção de cesarianas, representando no ano de 2016 (37,97\%). Mesmo assim, apresenta-se com a maior proporção em comparação com demais regiões no mesmo ano (Norte 11,78\%; Nordeste: 29,70\%; Centro-Oeste: 23,56\%).

Ao analisar a região Centro-Oeste, as mulheres brancas apresentaram a maior proporção de cesarianas, variando de 64,79\% em 2007 a 76,12\% em 2016 e as indígenas apresentou a menor proporção, com 16,05\% (2007) e 23,56\% (2016). As demais tiveram uma maior oscilação nas proporções ao longo dos anos, finalizando a série histórica com valores aproximados (preta: 55,81\%; amarela: 57,85\%; e parda: $59,06 \%)$. 
Tabela 2 - Proporção de cesarianas segundo cor da pele e região do país de 2007 a 2016.

\begin{tabular}{|c|c|c|c|c|c|c|c|c|c|c|}
\hline \multirow[b]{2}{*}{ Raça/Etnia } & \multicolumn{10}{|c|}{ Ano } \\
\hline & 2007 & 2008 & 2009 & 2010 & 2011 & 2012 & 2013 & 2014 & 2015 & 2016 \\
\hline \multicolumn{11}{|l|}{ Norte } \\
\hline Branca & 52,26 & 55,32 & 59,29 & 60,99 & 62,28 & 63,14 & 65,80 & 66,80 & 66,90 & 65,58 \\
\hline Preta & 23,66 & 29,53 & 28,10 & 33,43 & 35,33 & 42,16 & 43,97 & 45,68 & 45,96 & 46,48 \\
\hline Amarela & 32,73 & 36,69 & 35,37 & 32,32 & 40,30 & 53,53 & 62,25 & 53,57 & 57,08 & 56,81 \\
\hline Parda & 33,29 & 35,42 & 37,32 & 39,46 & 40,98 & 43,74 & 45,30 & 46,30 & 45,45 & 45,15 \\
\hline Indigena & 6,95 & 7,77 & 9,37 & 9.48 & 9,79 & 10,26 & 11,42 & 10,31 & 11,91 & 11,78 \\
\hline \multicolumn{11}{|l|}{ Nordeste } \\
\hline Branca & 48,71 & 53,50 & 56,34 & 60,43 & 60,67 & 63,09 & 65,04 & 65,04 & 64,60 & 64,78 \\
\hline Preta & 22,10 & 26,48 & 27,02 & 33,05 & 37,81 & 38,55 & 40,42 & 40,42 & 41,15 & 41,46 \\
\hline Amarela & 18,86 & 23,27 & 28,91 & 31,99 & 46,47 & 46,17 & 48,10 & 48,10 & 54,11 & 51,09 \\
\hline Parda & 32,73 & 35,17 & 37,29 & 40,43 & 43,08 & 45,88 & 47,45 & 47,45 & 47,38 & 47,82 \\
\hline Indigena & 16,71 & 14,46 & 16,56 & 19,91 & 25,40 & 26,55 & 27,43 & 27,43 & 29,55 & 29,70 \\
\hline \multicolumn{11}{|l|}{ Sudeste } \\
\hline Branca & 60,95 & 62,71 & 64,15 & 65,54 & 66,75 & 68,95 & 69,80 & 69,67 & 67,62 & 67,38 \\
\hline Preta & 41,79 & 44,62 & 45,53 & 47,04 & 50,53 & 52,53 & 53,88 & 54,38 & 52,39 & 52,19 \\
\hline Amarela & 59,74 & 58,86 & 55,31 & 59,14 & 61,64 & 60,23 & 60,30 & 60,62 & 59,32 & 59,73 \\
\hline Parda & 41,44 & 43,00 & 44,47 & 46,69 & 49,16 & 52,04 & 52,90 & 52,62 & 51,07 & 50,86 \\
\hline Indigena & 25,14 & 23,64 & 22,00 & 19,85 & 27,17 & 31,70 & 33,90 & 32,52 & 33,09 & 35,36 \\
\hline \multicolumn{11}{|l|}{ Sul } \\
\hline Branca & 53,91 & 55,60 & 57,07 & 59,23 & 61,36 & 63,75 & 65,12 & 64,75 & 62,70 & 62,85 \\
\hline Preta & 38,10 & 39,69 & 42,44 & 42,80 & 47,21 & 49,96 & 50,04 & 49,96 & 49,05 & 49,67 \\
\hline Amarela & 60,62 & 66,96 & 66,23 & 68,99 & 64,58 & 70,68 & 67,61 & 65,06 & 61,22 & 58,54 \\
\hline Parda & 40,33 & 43,06 & 44,84 & 46,32 & 50,65 & 53,07 & 53,74 & 54,59 & 52,50 & 52,90 \\
\hline Indigena & 22,81 & 28,02 & 27,40 & 31,96 & 31,39 & 38,47 & 41,20 & 39,37 & 38,37 & 37,97 \\
\hline \multicolumn{11}{|c|}{ Centro Oeste } \\
\hline Branca & 64,79 & 66,09 & 68,86 & 71,27 & 72,74 & 75,58 & 76,11 & 76,35 & 76,08 & 76,12 \\
\hline Preta & 40,25 & 40,25 & 40,26 & 48,96 & 50,63 & 52,59 & 54,40 & 53,13 & 55,14 & 55,81 \\
\hline Amarela & 46,79 & 50,37 & 57,70 & 54,74 & 63,30 & 67,37 & 62,37 & 62,20 & 60,09 & 57,85 \\
\hline Parda & 43,94 & 45,65 & 46,78 & 47,83 & 52,37 & 55,95 & 57,12 & 58,17 & 57,21 & 59,06 \\
\hline Indig̣ena & 16,05 & 13,47 & 15,58 & 15,65 & 16,74 & 18,43 & 18,43 & 20,49 & 21,03 & 23,56 \\
\hline
\end{tabular}

Fonte: Elaborado pelas pesquisadoras a partir de dados do Brasil, 2018.

\section{DISCUSSÃO}

Segundo as diretrizes de atenção à gestante (BRASIL, 2016), considera-se que a taxa de cesariana de referência, ajustada para a população brasileira, deveria ser de $25 \%$ a $30 \%$. Contudo, os resultados revelados no presente estudo indicam que o Brasil está distante de alcançar essa meta. Durante o período analisado houve aumento de $8,88 \%$ na proporção de cesarianas, sendo que no último ano da série, a taxa de cesariana correspondeu a 55,44\%, ou seja, aproximadamente o dobro do recomendado para o país, fato preocupante, visto que, taxas de cesariana acima de $10 \%$ não estão relacionadas à redução da mortalidade materna e neonatal e sem a indicação clínica podem estar relacionadas ao aumento de desfechos negativos na saúde (ORGANIZAÇÃO MUNDIAL DA SAÚDE, 2015; BRASIL, 2018; ENTRINGER et al., 2018).

Diversos fatores mostraram-se influentes para a elevada proporção de 
cesarianas no Brasil. Dentre eles: a região de residência e os cuidados do pré-natal; variáveis não clínicas, como a idade e a escolaridade da mulher, sua origem étnica; além da influência do profissional que a assiste no pré-natal e no parto. Dados nacionais e de outros países reforçam a ideia de que as características socioeconômicas da região de residência da mulher são mais determinantes nas proporções de cesarianas que os fatores socioeconômicos individuais e familiares das gestantes (MADEIRO; RUFINO; SANTOS, 2017).

O perfil das desigualdades sociais aponta para o paradoxo de que mulheres com piores condições socioeconômicas e, por consequência, maior risco de complicações no parto, têm menor acesso à cesariana do que aquelas com baixo risco e alto poder aquisitivo (BETRÁN et al., 2016). Ou seja, encontra-se maior frequência de cesarianas nas regiões mais ricas, com maior número de habitantes e de partos (MADEIRO; RUFINO; SANTOS, 2017). Nesse sentido, Hofelmann (2012), ao descrever a tendência temporal e a distribuição de cesarianas no Brasil e suas regiões no período de 1994 a 2009, observou uma tendência de aumento das cesarianas em todas as regiões, exceto a Centro-Oeste que se manteve alta e constante, com variações entre $32 \%$ a $48,4 \%$, do primeiro ao último período analisado, o que representa um incremento de $51,3 \%$ no período de 16 anos investigados. Já na série histórica analisada no presente artigo, a proporção de cesarianas variou de 46,56\%, para $55,44 \%$.

Da mesma forma, o estudo desenvolvido por Rattner e Moura (2016), ao descrever nascimentos via cesariana e vaginal e identificar associação com variáveis temporais e sociodemográficas, observaram que a região identificada com maior probabilidade e alta proporção de cesariana foi a Centro-Oeste, comparativamente à Norte com menor proporção, e as Regiões Nordeste, Sudeste e Sul apresentaram menor probabilidade, apesar das altas taxas nas duas últimas.

Madeiro, Rufino e Santos (2017), ao investigarem a tendência de cesarianas no Piauí de 2000 a 2011 constataram que, desde 2009, a proporção de cesarianas é superior aos partos vaginais, com elevação entre mulheres brancas com escolaridade maior ou igual a 12 anos de estudo. Corroborando com o exposto, os achados deste estudo apontam que mulheres brancas realizam mais cesarianas que as mulheres não brancas. Os resultados sinalizam também quanto maior o nível de escolaridade, maior a proporção de cesarianas realizadas, sendo as mulheres com 12 anos ou mais de estudo, residentes na região Centro-Oeste, a categoria com a maior proporção de cesarianas no país.

Em relação à cor da pele, apesar da predominância da cor branca sobre as demais, Barros et al. (1991) observaram maior prevalência de cesarianas entre mulheres de cor preta e menor entre mulheres pardas, diferente do presente estudo, no qual a proporção de cesarianas nas pardas supera à negra nas regiões Nordeste, Sul e Centro-Oeste, no ano de 2016. Para Madeiro, Rufino e Santos (2017), as 
disparidades em relação a cor da pele na proporção de cesáreas podem decorrer tanto de características socioculturais como de deficiências na assistência médica.

Neste estudo, a cor da pele com menor proporção de cesarianas em todas as regiões observadas foi a indígena, contudo, observa-se diferenças quanto à variação dessa proporção. A região Norte desponta com as menores proporções observadas, sendo as proporções de cesarianas em indígenas corresponderam a 6,95\% (2007) e $11,78 \%$ (2016), o que demonstra um crescimento de $4,83 \%$.

Já a região Sul apareceu como a região com maior proporção de cesarianas em indígenas, com uma proporção de 22,81\% (2007) e 37,97\% (2016), o que equivale a um crescimento de $15,16 \%$, ou seja, quase duas vezes maior que a proporção de crescimento na região Norte. Isso quer dizer que nem o fato de ser indígena protege a gestante da medicalização do parto, especialmente na região Sul, corroborando que as características locorregionais de residência da mãe parecem ter uma influência maior na realização de cesarianas do que características individuais e sociodemográficas maternas.

De acordo com esta pesquisa, houve um aumento expressivo na proporção de cesarianas em todas as regiões, escolaridades e cor da pele. Portanto não apenas o perfil de alta escolaridade e maior idade materna que historicamente apresentam altas taxas de cesariana cresceram, mas as demais categorias também ficaram acima das recomendações de cesariana da OMS (RATTNER; MOURA, 2016).

Assim, os resultados encontrados convergem com a literatura, que também verificaram associação positiva entre frequência de cesarianas e nível de escolaridade das mães (RATTNER; MOURA, 2016; FREITAS; FERNANDES, 2016; HOFELMANN, 2012; MENDOZA-SASSI et al., 2010; REBELO et al., 2010), e constataram que mulheres com mais escolaridade apresentam de três a quatro vezes mais probabilidade de realizar cesarianas quando comparados com mulheres analfabetas (BARROS et al., 1991; LIMWATTANANON; TANGCHAROENSATHIEN; SIRILAK, 2011).

Tal escolha pode estar relacionada ao fato de esse perfil de mulheres acreditar que a cesariana é mais segura, mais conveniente e menos dolorosa do que o parto vaginal e que a melhor qualidade da assistência ao parto estaria associada ao maior uso de tecnologias. Além disso, mulheres com mais escolaridade teriam, de uma forma geral, maior conhecimento e poder para solicitar um parto operatório, quando comparadas com mulheres com menor escolaridade (BARROS et al., 1991; MADEIRO; RUFINO; SANTOS, 2017). A escolha pela cesariana também pode ser explicada pelo medo e por fantasias em relação ao parto expressos durante a gestação, que podem ser agravados pelo fornecimento insuficiente de informações por parte da equipe e dos médicos durante o pré-natal (HASLINGER et al., 2011).

Importante destacar que a crença que correlaciona qualidade na assistência com 
o uso indiscriminado de tecnologias durante o parto não encontra amparo na literatura. Pelo contrário, o modelo de atenção a partos e nascimentos mais bem-sucedido e com melhores resultados perinatais é justamente aquele com menos intervenções. Nesse sentido, é necessário uma mudança de modelo de atenção à saúde no país, de forma a reduzir as muitas intervenções sem indicação clínica, com propostas mais ousadas na direção de limitar a ocorrência de cesarianas desnecessárias, as quais devem fazer parte das políticas públicas da área (LEAL et al., 2014; RATTNER; MOURA, 2016).

Uma dessas propostas, que visa diminuir a expressiva proporção de cesarianas ocorridas no setor privado, é a publicação da Resolução normativa no 368 da Agência Nacional de Saúde Suplementar (ANS) a qual dispõe, entre outras medidas, sobre o direito de acesso à informação das beneficiárias aos percentuais de cirurgias cesáreas e de partos normais, por operadora, por estabelecimento de saúde e por médico e sobre a utilização do partograma (BRASIL, 2015). Essa proposta pretende fomentar maior transparência por parte dos médicos quanto às indicações clínicas da cesárea, introduzindo informações a parturiente e até mesmo garantindo a proteção de seu direito de escolha.

Além disso, a Política Nacional de Humanização (PNH), que dedicou um de seus Cadernos para a Humanização do Parto e do Nascimento (BRASIL, 2014) é um outro exemplo de política pública desenvolvida no Brasil para enfrentar o desafio que os achados do presente estudo evidenciam. A concepção da $\mathrm{PNH}$, para Martins et al (2014), é de que é preciso intervir em práticas de saúde "desumanizadoras" que se encontram naturalizadas para qualificar o modelo de atenção e de gestão do parto e do nascimento. O que está em jogo é a transformação de um cenário em que nem sempre há articulação entre a assistência ao pré-natal oferecida pela Atenção Básica com a assistência ao parto que acontece na maternidade, instituição essa que muitas vezes a mulher nunca visitou. Dessa forma, se faz necessário fortalecer e ampliar a interlocução entre a rede de atenção, a fim de possibilitar um cuidado mais humanizado e integral à gestante e ao bebê.

Assim sendo, no presente estudo, a tendência de queda nas proporções de cesarianas observadas em algumas categorias nos anos finais da série histórica pode ser reflexo dos esforços empreendidos pelo Ministério da Saúde através da PNH e da ANS para a transformação dessa realidade. Da mesma forma, a gradativa e concomitante implementação da Rede Cegonha no âmbito do SUS, visando assegurar à mulher o direito ao planejamento reprodutivo e à atenção humanizada à gravidez, parto, puerpério e a garantia à criança ao nascimento seguro e desenvolvimento saudável, a partir de 2011, pode ter contribuído também com as recentes modificações na proporção de cesarianas (BRASIL, 2011). 


\section{CONSIDERAÇÕES FINAIS}

Os resultados encontrados no presente estudo apontam para um aumento gradativo na proporção de cesarianas realizadas de 2007 a 2016, com uma leve diminuição nos últimos dois anos (2015 e 2016). Pode-se observar uma proporção maior de cesarianas nas regiões Centro Oeste e Sul, em mulheres brancas e de alta escolaridade. Apesar disso, houve aumento da proporção de cesarianas em mulheres indígenas, principalmente na região Sul, o que aponta para uma determinação maior da região de residência da mãe em relação às demais variáveis como raça e escolaridade.

Considera-se necessário intensificar a implementação de políticas públicas de caráter intersetorial, envolvendo pesquisadores, sociedade, setores governamentais no Brasil e no mundo, voltadas à humanização no parto e no nascimento. Assim como, sugere-se que novos estudos sejam realizados com análises mais robustas, a fim de compreender melhor esse fenômeno.

Com a expansão das políticas aqui citadas, espera-se que a análise de futuras séries históricas apresente dados que comprovem uma reconfiguração do cenário nacional, com melhora na proporção entre os tipos de parto e uma real aproximação da meta estabelecida de $10 \%$ a $15 \%$ de cesarianas entre o total de partos.

Este estudo apresenta algumas limitações ao não analisar o número de consultas de pré-natal, a influência do médico na escolha do tipo de parto e o local de realização do parto (instituições públicas ou privadas); características consideradas importantes neste objeto de análise.

\section{REFERÊNCIAS}

BARROS, F. et al. Epidemic of caesarean sections in Brazil. The Lancet, v. 338, n. 8760, p. 167-169, 1991.

BETRAN, A. P. et al. The Increasing Trend in Caesarean Section Rates: Global, Regional and National Estimates: 1990-2014. PLoS ONE, San Francisco, v. 11, n. 2 , e0148343, 2016.

BOERMA, T. et al. Global epidemiology of use of and disparities in caesarean sections. The Lancet, v. 392, e10155, p.1341-8, 2018.

BRASIL. Ministério da Saúde. Departamento de Informática do SUS - DATASUS. Sistema de Informações sobre Nascidos Vivos - SINASC. Brasília, DF: Ministério da Saúde. 2018. Disponível em:

<http://tabnet.datasus.gov.br/cgi/deftohtm.exe?sinasc/cnv/nvuf.def>. Acesso em: 30 out. 2018

BRASIL. Ministério da Saúde. Diretrizes de atenção à gestante: a operação cesariana. Brasília, DF: Comissão Nacional de Incorporação de Tecnologias no SUS. 2016. Disponível em: 
<http://conitec.gov.br/images/Consultas/Relatorios/2015/Relatorio_PCDTCesariana_C P.pdf>. Acesso em: 30 out. 2018.

BRASIL. Ministério da Saúde. Humanização do parto e do nascimento. Brasília: Ministério da Saúde, 2014. 465 p. Disponível em:

<http://www.redehumanizasus.net/sites/default/files/caderno_humanizasus_v4_humani zacao_parto.pdf>. Acesso em: 27 fev. 2019.

BRASIL. Diretoria Colegiada da Agência Nacional de Saúde Suplementar - ANS.

Resolução Normativa - RN № 368, de 6 de janeiro de 2015. Dispõe sobre o direito de acesso à informação das beneficiárias aos percentuais de cirurgias cesáreas e de partos normais, por operadora, por estabelecimento de saúde e por médico e sobre a utilização do partograma, do cartão da gestante e da carta de informação à gestante no âmbito da saúde suplementar, 2015. Disponível em:

<http://www.ans.gov.br/component/legislacao/?view=legislacao\&task=TextoLei\&format =raw\&id=Mjg5Mg $>$. Acesso em: 27 fev. 2019.

BRASIL. Ministério da Saúde. Portaria № 1.459, de 24 de junho de 2011. Institui, no âmbito do Sistema Único de Saúde - SUS - a Rede Cegonha. Diário Oficial [da] República Federativa do Brasil, Brasília, DF, jun. Seção 1. 2011.

COSTA-RAMÓN, A. M. et al. It's about time: Cesarean sections and neonatal health. Journal of Health Economics. v. 59, p. 46-59, 2018.

ENTRINGER, A. P. et al. Impacto do planejamento do parto vaginal e da cesariana eletiva sem indicação clínica no Brasil. Rev Panam Salud Publica, Washington, v. 42, 116, 2018.

FREITAS, P. F.; FERNANDES, T.M. Associação entre fatores institucionais, perfil da assistência ao parto e as taxas de cesariana em Santa Catarina. Rev Bras Epidemiol, São Paulo, n. 19, n. 3, p. 525-538, 2016.

FUNDO DAS NAÇÕES UNIDAS PARA A INFÂNCIA (UNICEF). Quem espera, espera, 2017. Disponível em:

<https://www.unicef.org/brazil/pt/quem_espera_espera.pdf>. Acesso em: 30 out. 2018.

HASLINGER, C. et al. A importância da Psicologia no atendimento mães e pais na maternidade. In: JORNADA DE PESQUISA EM PSICOLOGIA: DESAFIOS ATUAIS NAS PRÁTICAS DA PSICOLOGIA, 4, 2011. Santa Cruz do Sul. Anais..., Santa Cruz do Sul: UNISC, 2011.

HOFELMANN, D. A. Tendência temporal de partos cesáreos no Brasil e suas Regiões: 1994 a 2009. Epidemiol. Serv. Saúde, Brasília, v. 21, n. 4, p. 561-568, 2012.

INSTITUTO BRASILEIRO DE GEOGRAFIA E ESTATÍSTICA - IBGE. Diretoria de Pesquisas Coordenação de População e Indicadores Sociais. Características étnicoraciais da população: um estudo das categorias de classificação de cor ou raça 2008. Rio de Janeiro: IBGE, 2011. p. 93. Disponível em: $<$ https://biblioteca.ibge.gov.br/visualizacao/livros/liv49891.pdf>. Acesso em: 20 fev. 2019.

LEAL, M. C. et al. Intervenções obstétricas durante o trabalho de parto e parto em mulheres brasileiras de risco habitual. Cadernos de Saúde Pública, Rio de Janeiro, v. 30, n. Suppl 1, p. 17-32, 2014. 
LINWATTANANON, S.; TANGCHAROENSATHIEN, V.; SIRILAK, S. Trends and inequities in where women delivered their babies in 25 low-income countries: evidence from Demographic and Health Surveys. Reprod Health Matters, v.19, n. 11, p. 75-85. 2011.

MADEIRO, A.; RUFINO, A. C.; SANTOS, A. O. Partos cesár,os no Piauí: tendência e fatores associados no período 2000-2011. Epidemiol. Serv. Saúde, Brasília, v. 26, n. 1, p.81-90, 2017.

MARTINS, C. P. et al. Humanização do parto e do nascimento: Pela gestação de formas de vida das quais possamos ser protagonistas. In: BRASIL. Ministério da Saúde. Humanização do parto e do nascimento. Ministério da Saúde, 2014. p. 9-18. Disponível em:

<http://www.redehumanizasus.net/sites/default/files/caderno_humanizasus_v4_humani zacao_parto.pdf>. Acesso em: 27 fev. 2019.

MENDOZA-SASSI, R. A. et al. Risk factors for cesarean section by category of health service. Rev Saúde Pública, São Paulo, v. 44, n. 1, p. 80-9, 2010.

ORGANIZAÇÃO MUNDIAL DE SAÚDE (OMS). Declaração da OMS sobre taxas de cesáreas. Genebra: OMS; 2015.

RATTNER, D.; MOURA, E. C. Nascimentos no Brasil: associação do tipo de parto com variáveis temporais e sociodemográficas. Rev. Bras. Saude Mater. Infant, Recife, v. 16, n. 1, p. 39-47, 2016.

REBELO, F. et al. High cesarean prevalence in a national population-based study in Brazil: the role of private practice. Acta Obstet Gynecol Scand, v. 89, n. 7, p. 903908, 2010.

RYAN, G. A.; NICHOLSON, S. M.; MORRISON, J. J. Vaginal birth after caesarean section: current status and where to from here? European Journal of Obstetrics and Gynecology and Reproductive Biology, v. 224, p. 52-57, 2018.

SANTA CATARINA. Secretaria de Estado da Saúde. Diretoria de Vigilância Epidemiológica - DIVE. Histórico do Sistema de Informações sobre Nascidos Vivos (SINASC). [internet]. 2018. Disponível em: <http://www.dive.sc.gov.br/index.php/historico-sinasc>. Acesso em: 30 out. 2018.

WHO. Global Survey on Maternal and Perinatal Health Research Group. Caesarean section without medical indications is associated with an increased risk of adverse short-term maternal outcomes, Organização Mundial da Saúde, 2010. Disponível em:

<http://apps.who.int/iris/bitstream/handle/10665/70494/WHO_RHR_HRP_10.20_eng.p df; jsessionid=2180DAF53F182CA93767A55D65870110? sequence=1 >. Acesso em: 03 nov. 2018. 